\title{
Radiomics in hepatic metastasis by colorectal cancer
}

\author{
Vincenza Granata', Roberta Fusco ${ }^{1 *}$, Maria Luisa Barretta', Carmine Picone ${ }^{1}$, Antonio Avallone ${ }^{2}$, Andrea Belli ${ }^{3}$, \\ Renato Patrone ${ }^{3}$, Marilina Ferrante ${ }^{4}$, Diletta Cozzi ${ }^{5}$, Roberta Grassi ${ }^{4}$, Roberto Grassi ${ }^{4}, 6$, Francesco Izzo ${ }^{3}$ and \\ Antonella Petrillo ${ }^{1}$
}

\begin{abstract}
Background: Radiomics is an emerging field and has a keen interest, especially in the oncology field. The process of a radiomics study consists of lesion segmentation, feature extraction, consistency analysis of features, feature selection, and model building. Manual segmentation is one of the most critical parts of radiomics. It can be timeconsuming and suffers from variability in tumor delineation, which leads to the reproducibility problem of calculating parameters and assessing spatial tumor heterogeneity, particularly in large or multiple tumors. Radiomic features provides data on tumor phenotype as well as cancer microenvironment. Radiomics derived parameters, when associated with other pertinent data and correlated with outcomes data, can produce accurate robust evidence based clinical decision support systems. The principal challenge is the optimal collection and integration of diverse multimodal data sources in a quantitative manner that delivers unambiguous clinical predictions that accurately and robustly enable outcome prediction as a function of the impending decisions.
\end{abstract}

Methods: The search covered the years from January 2010 to January 2021. The inclusion criterion was: clinical study evaluating radiomics of liver colorectal metastases. Exclusion criteria were studies with no sufficient reported data, case report, review or editorial letter.

Results: We recognized 38 studies that assessed radiomics in mCRC from January 2010 to January 2021. Twenty were on different tpics, 5 corresponded to most criteria; 3 are review, or letter to editors; so 10 articles were included.

Conclusions: In colorectal liver metastases radiomics should be a valid tool for the characterization of lesions, in the stratification of patients based on the risk of relapse after surgical treatment and in the prediction of response to chemotherapy treatment.

Keywords: Radiomics, Texture analysis, Liver metastases, Prognosis, Treatment assessment

\footnotetext{
* Correspondence: r.fusco@istitutotumori.na.it

'Radiology Division, "ISTITUTO NAZIONALE TUMORI - IRCCS - FONDAZIONE

G. PASCALE, Napoli, Italy", Via Mariano Semmola, Naples, Italy

Full list of author information is available at the end of the article
}

C C The Author(s). 2021 Open Access This article is licensed under a Creative Commons Attribution 4.0 International License, which permits use, sharing, adaptation, distribution and reproduction in any medium or format, as long as you give appropriate credit to the original author(s) and the source, provide a link to the Creative Commons licence, and indicate if changes were made. The images or other third party material in this article are included in the article's Creative Commons licence, unless indicated otherwise in a credit line to the material. If material is not included in the article's Creative Commons licence and your intended use is not permitted by statutory regulation or exceeds the permitted use, you will need to obtain permission directly from the copyright holder. To view a copy of this licence, visit http://creativecommons.org/licenses/by/4.0/. The Creative Commons Public Domain Dedication waiver (http://creativecommons.org/publicdomain/zero/1.0/) applies to the data made available in this article, unless otherwise stated in a credit line to the data. 


\section{Introduction}

Radiomics is an emerging field and has a keen interest, especially in the oncology field [1-4]. It has been shown that radiomics could be predictive of TNM grade, histological grade, response to therapy, and survival in various tumors [5-8]. The process of a radiomics study consists of lesion segmentation, feature extraction, consistency analysis of features, feature selection, and model building.

Manual segmentation is one of the most critical parts of radiomics. It can be time-consuming and suffers from variability in tumor delineation, which leads to the reproducibility problem of calculating parameters and assessing spatial tumor heterogeneity, particularly in large or multiple tumors $[9,10]$.

Radiomic features provides data on tumor phenotype as well as cancer microenvironment. Radiomics derived parameters, when associated with other pertinent data and correlated with outcomes data, can produce accurate robust evidence-based clinical-decision support systems (CDSS) [11]. The potential of radiomics to improve CDSS is beyond doubt and the field is evolving rapidly. The principal challenge is the optimal collection and integration of diverse multimodal data sources in a quantitative manner that delivers unambiguous clinical predictions that accurately and robustly enable outcome prediction as a function of the impending decisions [12]. The central hypothesis of radiomics is that the quantitative individual voxel-based variables are more sensitively associated with various clinical end points compared with the more qualitative radiologic, histo-pathologic, and clinical data more commonly used today [13]. Radiomic variables offer notable advantages over qualitative imaging assessment, since this is clearly limited by the resolution of observers' eyes. An extension of radiomic information can be accomplished by adding these data to existing prognostic tools, such as genomics. Genomics is an emerging prognostic tool; in fact, genomic markers, along with expression of various microRNA signatures, have been shown to correlate with treatment response, metastatic spread, and prognosis [14-16]. Hence, combining radiomics with genomic data, socalled "radio-genomics," could potentially provide the highest level of personalized risk stratification ever developed to further advance precision medicine [13]. Radiogenomic may be able to greatly augment patient selection for different cancer therapy, predicting response to treatment, addressing potential resistance to therapy (chemotherapy and/or radiation therapy), distinguishing favorable subsets of malignancies from those with poor prognosis, evaluating which patients may benefit from adjuvant therapy $[13,17]$.

Hard coded radiomic features were proven to be effective to predict the chemotherapy response in liver colorectal metastases (mCRC), which manifested their clinical usefulness in response prediction [18-20]. However, previously used hard-coded texture features were not specifically designed for targeted clinical issues, which limited their predictive validity. With the development of the deep learning (DL) technique, the neural network is more commonly used in radiomics studies, and has achieved expert-level performance in rectal cancer and liver diseases [21, 22]. DL self-learning quantitative features may supplement unrevealed imaging features besides conventional radiomic features to improve the predictive power. Additionally, DL-based radiomics avoided time-consuming [23].

This article is an update overview on the Radiomics in liver colorectal metastases. Particularly, limitations and future perspectives are discussed.

\section{Methods}

This overview is the result of a self-study without protocol and registration number.

\section{Search criterion}

We assessed several electronic databases: PubMed (US National Library of Medicine, http://www.ncbi.nlm.nih. gov/pubmed), Scopus (Elsevier, http://www.scopus.com/), Web of Science (Thomson Reuters, http://apps. webofknowledge.com/), and Google Scholar (https:// scholar.goo-gle.it/). The following search criteria have been used: "Radiomics" AND "Liver colorectal metastases" AND "detection"; "Radiomics" AND "Liver colorectal metastases" AND "diagnosis"; "Radiomics" AND "Liver colorectal metastases" AND "Characterization"; "Radiomics" AND "Liver colorectal metastases" AND "Prognosis"; "Radiomics" AND "Liver colorectal metastases" AND "treatment assessment".

The search covered the years from January 2010 to January 2021. Moreover, the references of the found papers were evaluated for publications not indexed in the electronic database. We analyzed all titles and abstracts. The inclusion criterion was: clinical study evaluating radiomics of liver colorectal metastases. Articles published in the English language from January 2010 to January 2021 were included. Exclusion criteria were studies with no sufficient reported data, case report, review or editorial letter.

\section{Results}

We recognized 38 studies that assessed Radiomics in mCRC from January 2010 to January 2021. Twenty papers were on different topics, 5 corresponds to most criteria; 3 are review, or letter to editors; so 10 articles were included in the analysis (Fig. 1). 


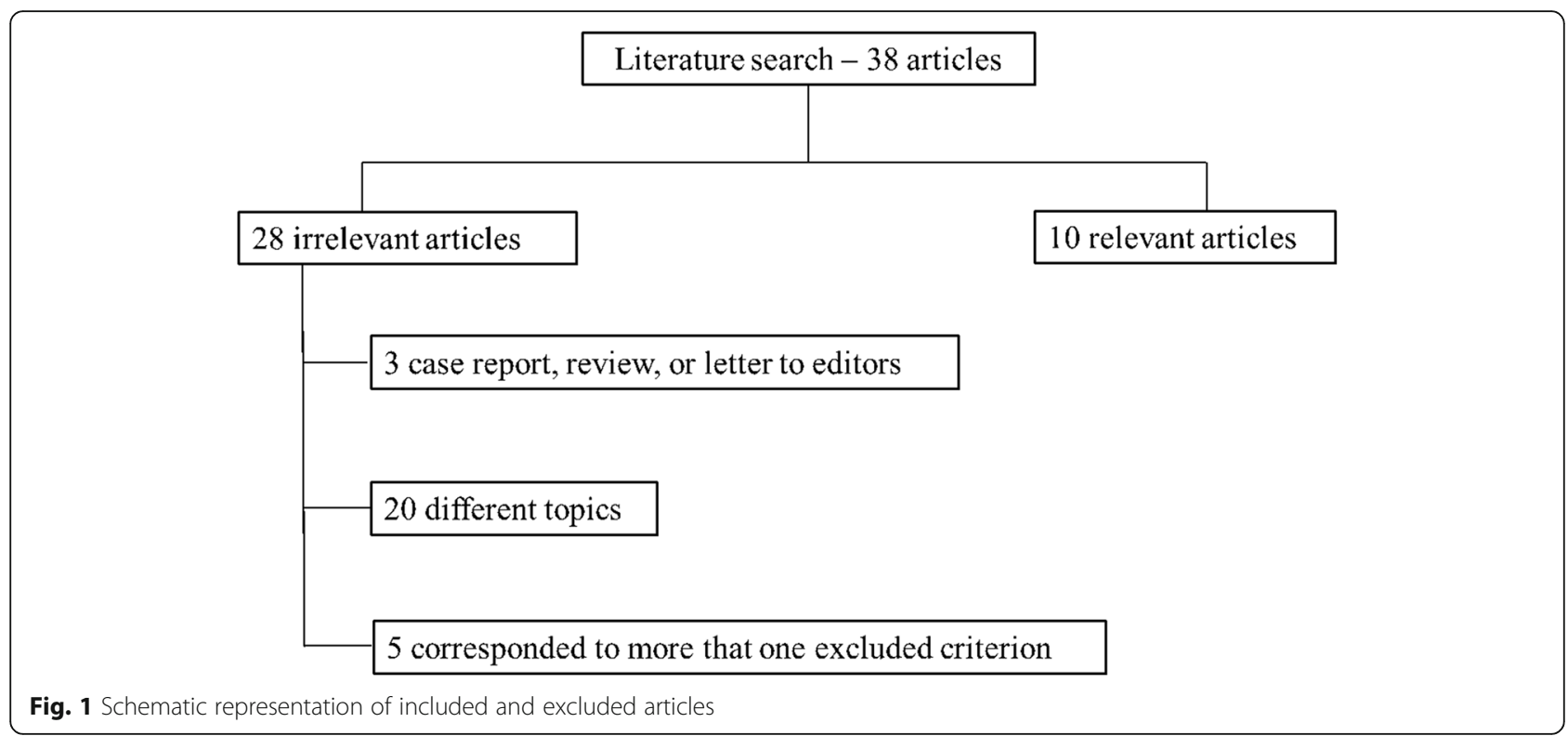

\section{Discussion}

\section{Basic principles and process}

Radiomics (Fig. 2) is simply the extraction of a high number of quantitative features from medical images, including several phases: image acquisition (e.g. RX, US, MX, CT, MRI, and ibrid imaging as PET-CT or PET$\mathrm{RM}$ ); segmentation (aumotatic, semi-automatic and manual); generation of features; development of database; analysis of database and radiomics signature [24]. Images used for radiomic analysis are collected from different hospitals or data centers; thus, these images are usually obtained using different parameters and protocols and reconstructed with different software. The differences may bring unexpected influences on the radiomic model. Segmentation is critical because the subsequent feature data are generated from the segmented volumes. It is challenging because many tumors have indistinct borders. Generation of features refers to the extraction of semantic features such as dimension, necrosis, margin, location or extraction of non semantic features such as shape, hystogram or texture [25-31].

In the past years, texture analysis has gained attention in medical applications and has been proved to be a significant computer-aided diagnostic tool [27]. There is not a strict definition of an image texture but it can be described as the spatial arrangement of patterns that provides the visual appearance of coarseness, randomness, smoothness, etc. Texture analysis describes a wide range of techniques for quantification of gray-level patterns and pixel inter-relationships within an image providing a measure of heterogeneity. It has been shown that different image areas exhibit different textural patterns that are sometimes imperceptible to the human eye [27]. Applications of texture analysis in medical imaging include classification and segmentation of tissues and lesions. Texture analysis applications involve a process that consists of six steps: image acquisition, region of interest (ROI) definition, ROI pre-processing,
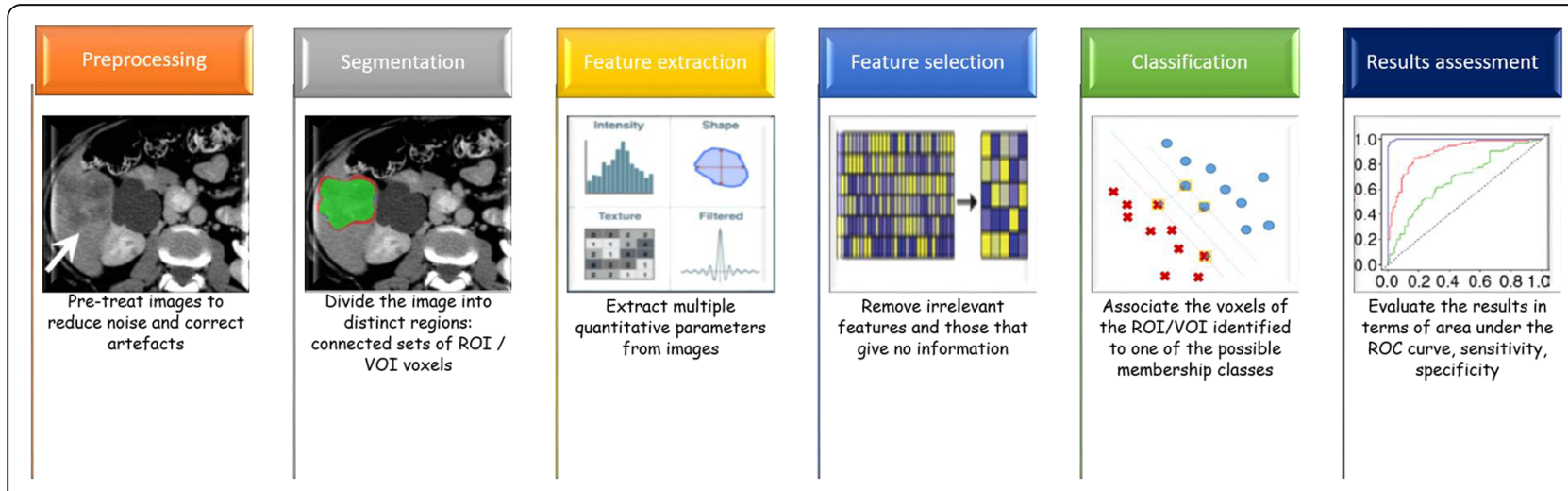

Fig. 2 Radiomic Workflow 
feature extraction, feature selection, and classification. None of these steps is specific, and the methods have to be chosen according to the application [27]. Manual definition of ROIs is still considered the gold standard in many applications, and it is the chosen option over automatic methods [27]. The size of the ROI should be sufficiently large to capture the texture information thereby eliciting statistical significance. The effect of ROI size becomes insignificant when large ROIs are used. In general, texture features were highly affected at ROI areas smaller than $80 \times 80$ pixels and became unaffected at ROI areas of around $180 \times 180$ pixels [27]. It was demonstrated that some features are not only dependent on texture, but also on other ROI properties, such as the mean intensity and variance. To avoid the influence of such factors, ROI normalization is a recommended pre-processing step [32, 33].

Feature extraction is the main and specific step in the texture analysis process and implies the computation of texture features from predefined ROIs (Fig. 3). Many approaches have been proposed in order to quantify the texture of an image allowing the computation of numerous features, including 2D methods (e.g. Model-based methods, Autoregressive models, Fractal models.) or 3D approaches [34]. 3D approaches increase the dimensionality and the information captured from the image, thus improving the discrimination power [35, 36]. Implementation of $4 \mathrm{D}$ texture analysis is possible by including the temporal dimension available in some MRI datasets. Notable results were observed for discrimination of benign and malignant breast lesions and for localization and segmentation of the heart using the 4D spatiotemporal approach [37].

Radiomics can be performed with as few as 100 patients, although larger data sets provide more power. Radiomics could potentially aid cancer detection, diagnosis, assessment of prognosis, prediction of response to treatment, monitoring of disease status [38, 39].

Radiomic is designed to be used in decision support of precision medicine, using standard of care images that are routinely acquired in clinical practice, it presents a cost-effective and highly feasible addition for clinical decision support. Also, this analysis non-invasively characterize the overall tumor accounting for heterogeneity, interrogating the entire tumor allows the expression of microscopic genomic and proteomics patterns in terms of macroscopic image-based features [39]. Moreover, Radiomics Analysis produces prognostic and/or predictive biomarker value derived from routine, standard of care imaging data, allowing for a fast, low-cost, and repeatable means for longitudinal monitoring [39].

\section{Current applications}

Radiomics approaches in diagnosis and assessment of prognosis

Studies using radiomic analyses have shown that radiomic features are capable of distinguishing between benign and malignant tissue and aiding in the assessment of cancer aggressiveness in a variety of clinical settings [40-43]. Analysis of heterogeneity in enhancement patterns or texture analysis of DCE images has been performed with promising findings; however, texture

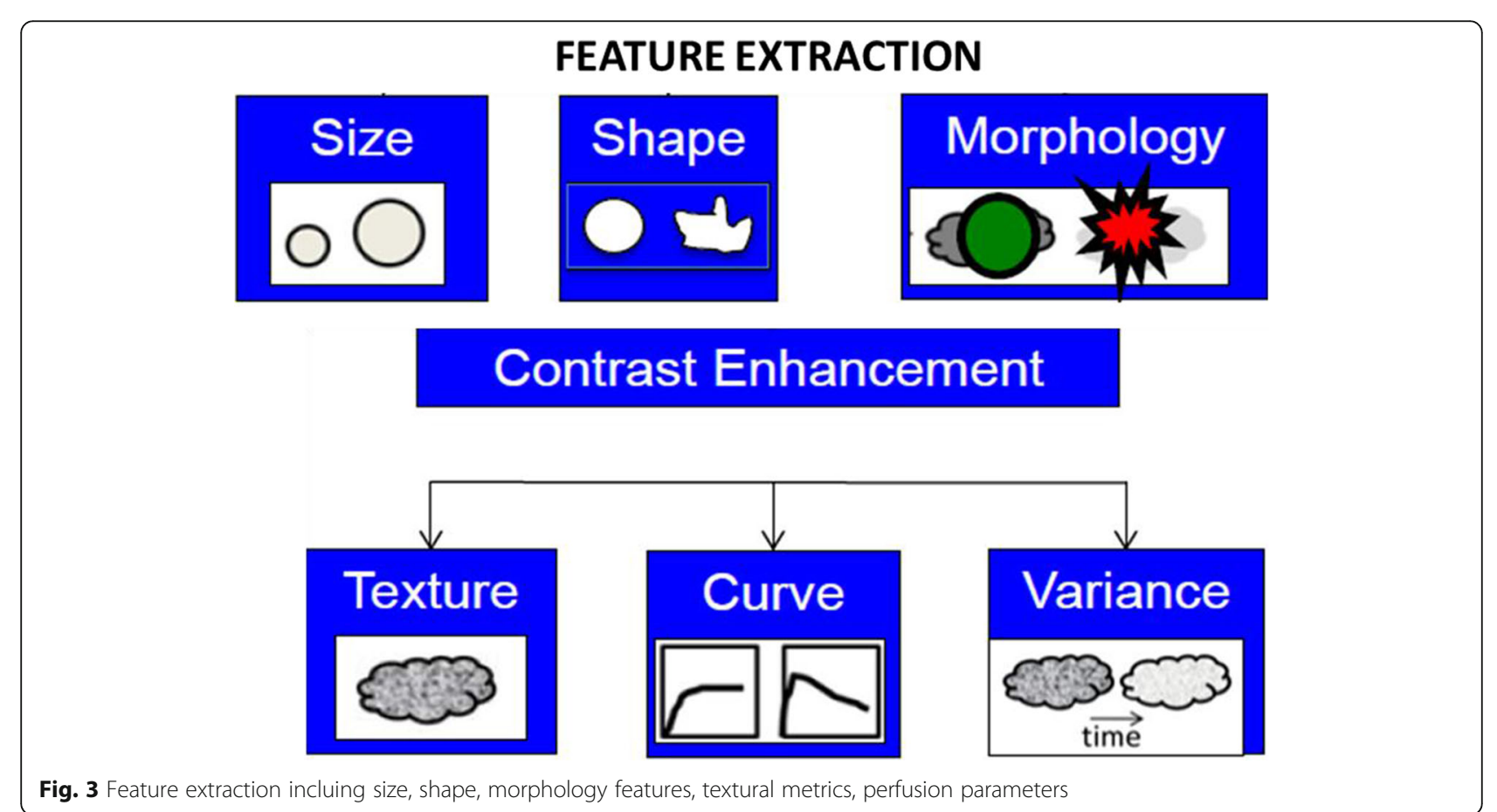


analysis is not limited to enhancement patterns. Measures of heterogeneity in T1- W, T2W, and DW MRI images can reveal differences in cellular density in tumours, which in turn can be matched to histological findings and aid in distinguishing malignant versus benign lesions $[40,43]$.

Oyama et al. assessed the accuracy for classification of hepatic tumors by characterization of T1-weighted magnetic resonance (MR) images using two radiomics approaches with machine learning models: texture analysis and topological data analysis using persistent homology. They showed that Texture features and persistence images have the potential to capture such arrays of gray scale values and might provide useful information for the differential diagnosis of hepatic tumors [44]. $\mathrm{Li}$ et al. assessed the feasibility of texture analysis based on spectral attenuated inversion-recovery (SPAIR) T2WMRI) for the classification of hepatic hemangioma $(\mathrm{HH})$, hepatic metastases (HM) and hepatocellular carcinoma (HCC). The texture analyses showed that the $\mathrm{HH}$ versus $\mathrm{HM}, \mathrm{HM}$ versus $\mathrm{HCC}$, and $\mathrm{HH}$ versus $\mathrm{HCC}$ could be differentiated by 9,16 and 10 feature parameters, respectively. The model's misclassification rates were 11.7, 9.6 and $9.7 \%$ respectively. No texture feature was able to adequately distinguish among the three types of single liver lesions at the same time [45]. Jansen et al. evaluated the MRI data sets of 95 patients with in total 125 benign lesions (40 adenomas, 29 cysts and 56 hemangiomas) and 88 malignant lesions (30 hepatocellular carcinomas (HCC) and 58 metastases). Contrast curve, gray level histogram, and gray level co-occurrence matrix texture features were extracted from the DCE-MR and T2weighted images. In addition, risk factors including the presence of steatosis, cirrhosis, and a known primary tumor were used as features. Fifty features with the highest ANOVA F- score were selected and fed to an extremely randomized trees classifier. The classifier evaluation was performed using the leave-one-out principle and receiver operating characteristic (ROC) curve analysis. They showed that the overall accuracy for the classification of the five major focal liver lesion types is 0.77 . The sensitivity/specificity is $0.80 / 0.78,0.93 / 0.93$, $0.84 / 0.82,0.73 / 0.56$, and $0.62 / 0.77$ for adenoma, cyst, hemangioma, HCC, and metastasis, respectively [46]. These data are similar to the data by Gatos et al. which reported an overall accuracy of 0.90 for the classification of benign, HCC and metastatic focal liver lesions using only T2-weighted MR images [47].

The prognostic and predictive value of radiomics in colorectal cancer metastases to the liver have been well studied, with several studies demonstrating the utility of diagnostic imaging in predicting clinical outcomes. Data from the current literature highlight the central role of both KRAS mutations as strong prognostic and predictive biomarkers among patients undergoing liver colorectal metastases resection (mCRC). In particular, mutations in $K R A S$ were strongly associated with worse overall survival (OS) and recurrence-free survival (RFS), as well as specific patterns of recurrence among patients with colorectal liver metastases $\mathrm{mCRC}$ [16]. The possibility to correlate radiomic parameters to KRAS status offers notable advantages over qualitative imaging assessment, allowing a better patient selection for cancer therapy, predicting response to treatment, distinguishing favorable subsets of patients from those with poor prognosis, evaluating which patients may benefit from surgical treatment. Moreover, a largely underappreciated aspect is the potential that radiomics may be just as pronounced in the economic realm because further optimization of patient selection and early recognition of toxicities undoubtedly influence the costeffectiveness of cancer care [16]. In our previous study, we assessed the association of RAS mutation status and radiomics-derived data by Contrast Enhanced-Magnetic Resonance Imaging (CE-MRI) in liver metastases. Texture metrics and parameters based on lesion morphology were calculated. Per-patient univariate and multivariate analysis were made. Wilcoxon-Mann-Whitney $U$ test, receiver operating characteristic (ROC) analysis, pattern recognition approaches with features selection approaches were considered. Significant results were obtained for texture features while morphological parameters had not significant results to classify RAS mutation. The results showed that using a univariate analysis was not possible to discriminate accurately the RAS mutation status. Instead, considering a multivariate analysis and classification approaches, a KNN exclusively with texture parameters as predictors reached the best results (AUC of 0.84 and an accuracy of $76.9 \%$ with $90.0 \%$ of sensitivity and $67.8 \%$ of specificity on training set and an accuracy of $87.5 \%$ with $91.7 \%$ of sensitivity and $83.3 \%$ of specificity on external validation cohort) [16]. Also Dercle et al. assessed the role of radiomics signature to predict tumor sensitiveness to irinotecan, 5fluorouracil, and leucovorin (FOLFIRI) alone (F) or in combination with cetuximab (FC) [48]. The signature (area under the ROC curve [95\% confidence interval (CI)]) used temporal decrease in tumor spatial heterogeneity plus boundary infiltration to successfully predict sensitivity to antiepidermal growth factor receptor therapy (FCHQ: 0.80 [95\% CI $=0.69$ to 0.94], FCSD: 0.72 [95\% $\mathrm{CI}=0.59$ to 0.83$]$ ) but failed with chemotherapy (FHQ: 0.59 [ $95 \% \mathrm{CI}=0.44$ to 0.72 ], FSD: 0.55 [ $95 \% \mathrm{CI}=0.43$ to $0.66])$. In cetuximab containing sets, radiomics signature outperformed existing biomarkers (KRAS-mutational status, and tumor shrinkage by RECIST 1.1) for detection of treatment sensitivity and was strongly associated with OS (two-sided $P<.005$ ) [48]. In an analysis of 77 mCRC patients, Lubner et al. investigated tumor heterogeneity through analysis of several quantitative texture parameters, 
including entropy, skewness, mean positive pixels, and standard deviation [49]. Correlations between these imaging parameters and patients clinico-pathologic features demonstrated an association between less entropy, standard deviation, and a higher mean positive pixel value with tumor grade. Furthermore, the degree of skewness was negatively correlated with KRAS mutations, and entropy was associated with OS [49]. These results were replicated in a retrospective study of 198 preoperative CT scans for patients undergoing resection of colorectal liver metastases [50].

An interesting field of application of radiomics is related to the assessment of lesions before the lesions became detectable. In a pre-clinical study Becker et al. [51] investigate whether any texture features show a correlation with intrahepatic tumor growth before the metastasis is visible to the human eye. Texture analysis was performed on the images yielding 32 texture features derived from histogram, gray level co-occurrence matrix, gray level run length matrix, and gray level size zone matrix. The features were examined with a linear regression model/Pearson correlation test and hierarchical cluster analysis. From each cluster, the feature with the lowest variance was selected. The research showed that texture features may quantitatively detect liver metastases before they become visually detectable by the radiologist.

\section{Assessment treatment}

Radiomics analysis has also demonstrated its potential in assessing treatment response for $\mathrm{mCRC}$ patients. In a study of 21 patients, Rao et al. compared CT texture analysis to Response Evaluation Criteria in Solid Tumors (RECIST) for assessing response to chemotherapy. Interestingly, they found that quantitative changes in entropy and uniformity were better at differentiating between good and poor responses when compared to changes in size or volume when using the RECIST criteria [51]. These results suggest that texture analysis may be better at predicting treatment response when compared to conventional size criteria, further expanding the utility of diagnostic imaging. MRI-based texture analysis has also been used to analyze treatment response to Yttrium-90 radioembolization in patients with liver metastases. In a retrospective cohort of 37 patients who underwent radioembolization, serial imaging based upon texture analysis and RECIST criteria monitored treatment response. The researchers showed that in patients with progressive disease, texture analysis was able to detect progression an average of 3.5 months before RECIST [52]. These findings, in addition to the ones previously addressed, demonstrate the diagnostic, prognostic, and therapeutic implications of imaging features, emphasizing their potential to significantly affect liver cancer outcomes [53-58].

\section{Current limitations}

At present, research on radiomics is still in its infancy and there are no standardized and unified standards for the complicated research process.

Radiomics features contain characteristics of both imaging and numeric features. Radiomics features generally refer to "agnostic" quantitative measurements that are mathematically extracted and differ from "semantic" features such as those covered by radiological lexicons $[59,60]$. Four main radiomics phenotypes have been used to capture tissue heterogeneity: 1) volume and shape; 2) first-order.

statistics to assess voxel distributions without considering their spatial relationship; 3) second-order statistics (texture analysis) to study spatial relationships among voxels; and 4) transformed features [61, 62].

Similar to common imaging biomarkers, the reproducibility of radiomics features can be questioned due to the nature of the imaging data itself. For example, intraindividual test-retest repeatability, image-acquisition technique, multi-machine reproducibility, and image reconstruction parameters all contribute in challenging reproducible research in radiomics. Another major challenge is imposed by the variations among the different techniques to process the images into analyzable quantitative data. One can obtain widely different results from the same radiomics data by using different transformation or feature-selection methods. With all these variations in image acquisition and processing in radiomics, it seems a daunting task to obtain a stable, generalizable result that can be consistently reproduced. Therefore, the reproducibility of radiomics features and modeling can be easily challenged, and great effort should be made to reduce variations [63-69].

Although most studies report rigorously quality-controlled manual segmentation, fully automated segmentation algorithms should be implemented for achieving standardization. Another important issue in several studies is the absence of clear identification of causes for false-positive results to further improve the capabilities of Convolutional neural network. In addition, for the selection of ROI, there is currently no suitable algorithm to calibrate tumor regions. Most studies calibrated ROI by radiologists, which increases the amount of pre-work, while calibration by different people will have an impact on the subsequent establishment of the model, leading to limited reproducibility of the results and comparability between studies [65]. In addition, a lack of standardization in reporting the results of research often makes it confusing for readers. We propose that future studies should report features based on the 'Image biomarker standardisation initiative' using formal nomenclature and corner marks. Furthermore, traditional machine-learning algorithms such as random forests and deep- learning 
algorithms like the neural network that have emerged in recent years can both be used for the establishment of radio- mics models. The algorithms used by each type of research are different. Still, there is no research to prove which algorithms are the most suitable for such work. Finally, most of the current research results are still in the training sample stage, so the high accuracy of the model does not reflect its actual predictive ability. Whether the model is effective or not depends on the validation phase by the test sample [65].

Current published studies rely on the data obtained from cohort with limited sample size. These databases should be made with clearly defined criteria, including definite histopathological diagnosis, genomic details, and large amount of clinical and biological data of patients.

\section{Conclusion}

In conclusion, while initial studies looking at radiomics have been very promising, there has been poor standardization and generalization of radiomic results, which limit the translation of this approach into clinical practice. Clear limitations of this field are emerging, especially with regard to data-quality control, repeatability, reproducibility, generalizability of results, and issues related to model overfitting. To address those problems, we propose that future radiomic research should be assessed via the radiomics quality score. By doing so, radiomics studies can be more comparable and increase its potential to be applied in future clinical practice, so that the advance in radiomics will largely contribute to the development of personalization and precision medicine.

\section{Acknowledgements}

The authors are grateful to Alessandra Trocino, librarian at the National Cancer Institute of Naples, Italy.

\section{Authors' contributions}

Each author has participated sufficiently in any submission to take public responsibility for its content. The authors read and approved the final manuscript.

\section{Funding}

No funding.

\section{Availability of data and materials}

Data sharing not applicable to this article as no datasets were generated or analysed during the current study.

\section{Declarations}

Ethics approval and consent to participate Not applicable.

\section{Consent for publication}

Not applicable.

\section{Competing interests}

The authors have no conflict of interest to be disclosed.

\section{Author details}

"Radiology Division, "ISTITUTO NAZIONALE TUMORI - IRCCS - FONDAZIONE G. PASCALE, Napoli, Italy", Via Mariano Semmola, Naples, Italy. ${ }^{2}$ Abdominal Oncology Division, "ISTITUTO NAZIONALE TUMORI - IRCCS - FONDAZIONE G. PASCALE, NAPOLI, ITALIA", Via Mariano Semmola, Naples, Italy. ${ }^{3}$ Hepatobiliary Surgical Oncology Division, "ISTITUTO NAZIONALE TUMORI - IRCCS FONDAZIONE G. PASCALE, NAPOLI, ITALIA", Via Mariano Semmola, Naples, Italy. ${ }^{4}$ Division of Radiology, "Università degli Studi della Campania Luigi Vanvitelli", Naples, Italy. ${ }^{5}$ Division of Radiology, "Azienda Ospedaliera Universitaria Careggi", Florence, Italy. ${ }^{6}$ Italian Society of Medical and Interventional Radiology SIRM, SIRM Foundation, Via della Signora 2, 20122 Milan, Italy.

Received: 29 March 2021 Accepted: 12 May 2021

Published online: 02 June 2021

\section{References}

1. Lambin P, Leijenaar RTH, Deist TM, Peerlings J, de Jong EEC, van Timmeren J, et al. Radiomics: the bridge between medical imaging and personalized medicine. Nat Rev Clin Oncol. 2017 Dec;14(12):749-62. https://doi.org/10.1 038/nrclinonc.2017.141.

2. Limkin EJ, Sun R, Dercle L, Zacharaki El, Robert C, Reuzé S, et al. Promises and challenges for the implementation of computational medical imaging (radiomics) in oncology. Ann Oncol. 2017 Jun 1;28(6):1191-206. https://doi. org/10.1093/annonc/mdx034.

3. Verma V, Simone CB 2nd, Krishnan S, Lin SH, Yang J, Hahn SM. The Rise of Radiomics and Implications for Oncologic Management. J Natl Cancer Inst. 2017;109(7). https://doi.org/10.1093/jnci/djx055.

4. Calin GA, Croce CM. MicroRNA signatures in human cancers. Nat Rev Cancer. 2006;6(11):857-66. https://doi.org/10.1038/nrc1997.

5. Campbell PJ, Yachida S, Mudie L, Stephens PJ, Pleasance ED, Stebbings LA, et al. The patterns and dynamics of genomic instability in metastatic pancreatic cancer. Nature. 2010;467(7319):1109-13. https://doi.org/10.1038/ nature09460.

6. Benedetti G, Mori M, Panzeri MM, Barbera M, Palumbo D, Sini C, et al. CTderived radiomic features to discriminate histologic characteristics of pancreatic neuroendocrine tumors. Radiol Med. 2021 Feb 1. https://doi. org/10.1007/s11547-021-01333-z.

7. Verma V, Lautenschlaeger T. MicroRNAs in non-small cell lung cancer invaSion and metastasis: from the perspective of the radiation oncologist. Expert Rev Anticancer Ther. 2016;16(7):767-74. https://doi.org/10.1080/1473 7140.2016.1191950.

8. Shi L, He Y, Yuan Z, Benedict S, Valicenti R, Qiu J, et al. Radiomics for response and outcome assessment for non-small cell lung Cancer. Technol Cancer Res Treat. 2018;17:1533033818782788.

9. Agazzi GM, Ravanelli M, Roca E, Medicina D, Balzarini P, Pessina C, et al. CT texture analysis for prediction of EGFR mutational status and ALK rearrangement in patients with non-small cell lung cancer. Radiol Med. 2021 Jan 29. https://doi.org/10.1007/s11547-020-01323-7.

10. Santone A, Brunese MC, Donnarumma F, Guerriero P, Mercaldo F, Reginelli A, et al. Radiomic features for prostate cancer grade detection through formal verification. Radiol Med. 2021 Jan 4;126(5):688-97. https://doi.org/1 0.1007/s11547-020-01314-8.

11. Alberich-Bayarri Á, Hernández-Navarro R, Ruiz-Martínez E, García-Castro F, García-Juan D, Martí-Bonmatí L. Development of imaging biomarkers and generation of big data. Radiol Med. 2017 Jun;122(6):444-8. https://doi.org/1 0.1007/s11547-017-0742-x.

12. Kirienko M, Ninatti G, Cozzi L, Voulaz E, Gennaro N, Barajon I, et al. Computed tomography (CT)-derived radiomic features differentiate prevascular mediastinum masses as thymic neoplasms versus lymphomas. Radiol Med. 2020 Oct;125(10):951-60. https://doi.org/10.1007/s11547-02001188-w.

13. Abdollahi H, Mofid B, Shiri I, Razzaghdoust A, Saadipoor A, Mahdavi A, et al. Machine learning-based radiomic models to predict intensity-modulated radiation therapy response, Gleason score and stage in prostate cancer. Radiol Med. 2019 Jun;124(6):555-67. https://doi.org/10.1007/s11547-0180966-4.

14. Zhang Y, Zhu Y, Zhang K, Liu Y, Cui J, Tao J, et al. Invasive ductal breast cancer: preoperative predict Ki-67 index based on radiomics of ADC maps. Radiol Med. 2020 Feb;125(2):109-16. https://doi.org/10.1007/ s11547-019-01100-1. 
15. Zhang L, Kang L, Li G, Zhang X, Ren J, Shi Z, et al. Computed tomographybased radiomics model for discriminating the risk stratification of gastrointestinal stromal tumors. Radiol Med. 2020 May;125(5):465-73. https://doi.org/10.1007/s11547-020-01138-6.

16. Granata V, Fusco R, Avallone A, De Stefano A, Ottaiano A, Sbordone C, et al. Radiomics-derived data by contrast enhanced magnetic resonance in RAS mutations detection in colorectal liver metastases. Cancers (Basel). 2021 Jan 25;13(3):453. https://doi.org/10.3390/cancers13030453.

17. Fusco R, Granata V, Petrillo A. Introduction to special issue of radiology and imaging of Cancer. Cancers (Basel). 2020 Sep 18;12(9):2665. https://doi.org/1 0.3390/cancers 12092665

18. Jia TY, Xiong JF, Li XY, Yu W, Xu ZY, Cai XW, et al. Identifying EGFR mutations in lung adenocarcinoma by noninvasive imaging using radiomics features and random forest modeling. Eur Radiol. 2019 Sep;29(9):4742-50. https://doi.org/10.1007/s00330-019-06024-y.

19. Yin Q, Hung SC, Rathmell WK, Shen L, Wang L, Lin W, et al. Integrative radiomics expression predicts molecular subtypes of primary clear cell renal cell carcinoma. Clin Radiol. 2018 Sep;73(9):782-91. https://doi.org/10.1016/j. crad.2018.04.009.

20. lannarelli A, Sacconi B, Tomei F, Anile M, Longo F, Bezzi M, et al. Analysis of $C T$ features and quantitative texture analysis in patients with thymic tumors: correlation with grading and staging. Radiol Med. 2018 May;123(5):345-50. https://doi.org/10.1007/s1 1547-017-0845-4

21. Granata V, Fusco R, Risi C, Ottaiano A, Avallone A, De Stefano A, et al. Diffusion-weighted MRI and diffusion kurtosis imaging to detect RAS mutation in colorectal liver metastasis. Cancers (Basel). 2020 Aug 26;12(9): 2420. https://doi.org/10.3390/cancers12092420.

22. Cusumano D, Meijer G, Lenkowicz J, Chiloiro G, Boldrini L, Masciocchi C, et al. A field strength independent MR radiomics model to predict pathological complete response in locally advanced rectal cancer. Radiol Med. 2020;126(3):421-9. https://doi.org/10.1007/s11547-020-01266-z.

23. Wei J, Cheng J, Gu D, Chai F, Hong N, Wang Y, et al. Deep learning-based radiomics predicts response to chemotherapy in colorectal liver metastases. Med Phys. 2020;48(1):513-22. https://doi.org/10.1002/mp.14563.

24. Reginelli A, Capasso R, Petrillo M, Rossi C, Faella P, Grassi R, et al. Looking for Lepidic component inside invasive adenocarcinomas appearing as $\mathrm{CT}$ solid solitary pulmonary nodules (SPNs): CT Morpho-Densitometric features and 18-FDG PET findings. Biomed Res Int. 2019;2019:7683648-9. https://doi.org/1 $0.1155 / 2019 / 7683648$.

25. Granata V, Grassi R, Fusco R, Izzo F, Brunese L, Delrio P, et al. Current status on response to treatment in locally advanced rectal cancer: what the radiologist should know. Eur Rev Med Pharmacol Sci. 2020;24(23):12050-62. https://doi.org/10.26355/eurrev_202012.

26. Petrillo A, Fusco R, Vallone $P$, Filice $S$, Granata V, Petrosino $T$, et al. Digital breast tomosynthesis and contrast-enhanced dual-energy digital mammography alone and in combination compared to $2 \mathrm{D}$ digital synthetized mammography and MR imaging in breast cancer detection and classification. Breast J. 2020 May;26(5):860-72. https://doi.org/1 $0.1111 /$ tbj.13739.

27. Fusco R, Granata V, Maio F, Sansone M, Petrillo A. Textural radiomic features and time-intensity curve data analysis by dynamic contrast-enhanced MRI for early prediction of breast cancer therapy response: preliminary data. Eur Radiol Exp. 2020;4(1):8. https://doi.org/10.1186/s41747-019-0141-2.

28. Grassi R, Belfiore MP, Montanelli A, Patelli G, Urraro F, Giacobbe G, et al. COVID-19 pneumonia: computer-aided quantification of healthy lung parenchyma, emphysema, ground glass and consolidation on chest computed tomography (CT). Radiol Med. 2020;18(4):1-8. https://doi.org/10.1 007/s11547-020-01305-9.

29. Grassi R, Cappabianca S, Urraro F, Feragalli B, Montanelli A, Patelli G, et al. Chest $C T$ computerized aided quantification of PNEUMONIA lesions in COVID-19 infection: a comparison among three commercial software. Int J Environ Res Public Health. 2020 Sep 22;17(18):6914. https://doi.org/10.3390/ ijerph17186914.

30. Patella F, Sansone M, Franceschelli G, Tofanelli L, Petrillo M, Fusco M, et al. Quantification of heterogeneity to classify benign parotid tumors: a feasibility study on most frequent histotypes. Future Oncol. 2020 Apr;16(12): 763-78. https://doi.org/10.2217/fon-2019-0736.

31. Fusco R, Raiano N, Raiano C, Maio F, Vallone P, Mattace Raso M, et al. Evaluation of average glandular dose and investigation of the relationship with compressed breast thickness in dual energy contrast enhanced digital mammography and digital breast tomosynthesis. Eur J Radiol. 2020 May; 126:108912. https://doi.org/10.1016/.jejrad.2020.108912.

32. Mayerhoefer ME, Szomolanyi P, Jirak D, Berg A, Materka A, Dirisamer A, et al. Effects of magnetic resonance image interpolation on the results of texturebased pattern classification: A phantom study. Investig Radiol. 2009;44(7): 405-11. https://doi.org/10.1097/RLI.0b013e3181a50a66.

33. Materka A, Strzelecki M, Lerski R, Schad L. Evaluation of texture features of test objects for magnetic resonance imaging. In: Pietikainen $\mathrm{M}$, editor. Infotech Oulu Workshop on Texture Analysis in Machine Vision. Infotech, Oulu, Finland; 1999. p. 13-9.

34. Materka A, Strzelecki M. Texture Analysis Methods:AReview. Institute of Electronics, Technical University of Lodz, Poland, vol. 11; 1998. p. 1-32.

35. Kovalev VA, Krugge F, Gertz HJ, Von Cramon DY. Three-dimensional texture analysis of MRI brain datasets. IEEE Trans Med Imaging. 2001;20(5):424-33. https://doi.org/10.1109/42.925295.

36. Mahmoud-Ghoneim D, Toussaint G, Constans JM, JD DC. Threedimensional texture analysis in MRI: a preliminary evaluation in gliomas. Magn Reson Imaging. 2003;21(9):983-7. https://doi.org/10.1016/50730-725X(03)00201-7.

37. Woods BJ, Clymer BD, Kurc T, Heverhagen JT, Stevens R, Orsdemir A, et al. Malignant-lesion segmentation using $4 \mathrm{D}$ co-occurrence texture analysis applied to dynamic contrast-enhanced magnetic resonance breast image data. J Magn Reson Imaging. 2007;25(3):495-501. https://doi.org/10.1002/ jmri.20837.

38. Nakata N. Recent technical development of artificial intelligence for diagnostic medical imaging. Jpn J Radiol. 2019;37(2):103-8. https://doi.org/1 0.1007/s11604-018-0804-6.

39. Simpson AL, Antonelli M, Bakas S, et al (2019) A large annotated medical image dataset for the development and evaluation of seg- mentation algorithms. arXiv; published online Feb 25. http://arxiv.org/abs/1902.09063.

40. Chen CK, Wu HT, Chiou HJ, et al. Differentiating benign and malignant soft tissue masses by magnetic resonance imaging: role of tissue component analysis. J Chin Med Assoc. 2009;72(4):194e201.

41. Fehr $\mathrm{D}$, Veeraraghavan $\mathrm{H}$, Wibmer $\mathrm{A}$, et al. Automatic classification of prostate cancer Gleason scores from multiparametric magnetic reso- nance images. Proc Natl Acad Sci U S A. 2015;112(46):E6265e73.

42. Aerts HJ, Velazquez ER, Leijenaar RT, et al. Decoding tumour phenotype by noninvasive imaging using a quantitative radiomics approach. Nat Commun. 2014:5(1):4006. https://doi.org/10.1038/ncomms5006.

43. Zhou M, Hall L, Goldgof D, et al. Radiologically defined ecological dynamics and clinical outcomes in glioblastoma multiforme: preliminary results. Translat Oncol. 2014;7(1):5e13.

44. Oyama A, Hiraoka Y, Obayashi I, Saikawa Y, Furui S, Shiraishi K, et al. Hepatic tumor classification using texture and topology analysis of non-contrastenhanced three-dimensional T1-weighted MR images with a radiomics approach. Sci Rep. 2019;9(1):8764. https:/doi.org/10.1038/s41598-019-45283-Z.

45. Li Z, Mao Y, Huang W, Li H, Zhu J, Li W, et al. Texture-based classification of different single liver lesion based on SPAIR T2W MRI images. BMC Med Imaging. 2017 Jul 13;17(1):42. https://doi.org/10.1186/s12880-017-0212-X.

46. Jansen MJA, Kuijf HJ, Veldhuis WB, Wessels FJ, Viergever MA, Pluim JPW. Automatic classification of focal liver lesions based on MRI and risk factors. PLoS One. 2019 May 16;14(5):e0217053. https://doi.org/10.1371/journal.pone. 0217053.

47. Gatos I, Tsantis S, Karamesini M, Spiliopoulos S, Karnabatidis D, Hazle JD, et al. Focal liver lesions segmentation and classification in nonenhanced T2weighted MRI. Med Phys. 2017:44(3):3695-705. https://doi.org/10.1002/ mp.12291.

48. Dercle L, Lu L, Schwartz LH, Qian M, Tejpar S, Eggleton P, et al. Radiomics Response Signature for Identification of Metastatic Colorectal Cancer Sensitive to Therapies Targeting EGFR Pathway. J Natl Cancer Inst. 2020; 112(9):902-12. https://doi.org/10.1093/jnci/djaa017 PMID: 32016387; PMCID: PMC7492770.

49. Lubner MG, Stabo N, Lubner SJ, del Rio AM, Song C, Halberg RB, et al. CT textural analysis of hepatic metastatic colorectal cancer: pre-treatment tumor heterogeneity correlates with pathology and clinical outcomes. Abdom Imaging. 2015 Oct;40(7):2331-7. https://doi.org/10.1007/s00261-0150438-4.

50. Simpson AL, Doussot A, Creasy JM, Adams LB, Allen PJ, DeMatteo RP, et al. Computed tomography image texture: a noninvasive prognostic marker of hepatic recurrence after hepatectomy for metastatic colorectal Cancer. Ann Surg Oncol. 2017;24(9):2482-90. https://doi.org/10.1245/s10434-017-5896-1. 
51. Becker AS, Schneider MA, Wurnig MC, Wagner M, Clavien PA, Boss A. Radiomics of liver MRI predict metastases in mice. Eur Radiol Exp. 2018;2(1): 11. https://doi.org/10.1186/s41747-018-0044-7.

52. Rao SX, Lambregts DM, Schnerr RS, Beckers RC, Maas M, Albarello F, et al. CT texture analysis in colorectal liver metastases: a better way than size and volume measurements to assess response to chemotherapy? United European Gastroenterol J. 2016;4(2):257-63. https://doi.org/10.1177/205064 0615601603.

53. Reimer RP, Reimer P, Mahnken AH. Assessment of therapy response to Transarterial Radioembolization for liver metastases by means of posttreatment MRI-based texture analysis. Cardiovasc Intervent Radiol. 2018 Oct; 41(10):1545-56. https://doi.org/10.1007/s00270-018-2004-2.

54. Wesdorp NJ, van Goor VJ, Kemna R, Jansma EP, van Waesberghe JHTM, Swijnenburg RJ, Punt CJA, Huiskens J, Kazemier G. Advanced image analytics predicting clinical outcomes in patients with colorectal liver metastases: a systematic review of the literature. Surg Oncol 202;38:101578. doi: https://doi.org/10.1016/j.suronc.2021.101578. Epub ahead of print. PMID: 33866191

55. Neri E, Coppola F, Miele V, Bibbolino C, Grassi R. Artificial intelligence: who is responsible for the diagnosis? Radiol Med. 2020;125(6):517-21. https://doi. org/10.1007/s11547-020-01135-9 Epub 2020 Jan 31. PMID: 32006241.

56. Citone M, Fanelli F, Falcone G, Mondaini F, Cozzi D, Miele V. A closer look to the new frontier of artificial intelligence in the percutaneous treatment of primary lesions of the liver. Med Oncol. 2020;37(6):55. https://doi.org/10.1 007/s12032-020-01380-y.

57. Granata V, Caruso D, Grassi R, Cappabianca S, Reginelli A, Rizzati R, et al. Structured reporting of rectal Cancer staging and restaging: a consensus proposal. Cancers (Basel). 2021;13(9):2135. https://doi.org/10.3390/cancers13 092135.

58. Patrone R, Granata V, Belli A, Palaia R, Albino V, Piccirillo M, et al. The safety and efficacy of Glubran 2 as biliostatic agent in liver resection. Infect Agent Cancer. 2021;16(1):19. https://doi.org/10.1186/s13027-021-00358-3 PMID: 33726798; PMCID: PMC7968309.

59. Park JE, Park SY, Kim HJ, Kim HS. Reproducibility and Generalizability in Radiomics Modeling: Possible Strategies in Radiologic and Statistical Perspectives. Korean J Radiol. 2019;20(7):1124-37. https://doi.org/10.3348/ kjr.2018.0070.

60. Cellina M, Pirovano M, Ciocca M, Gibelli D, Floridi C, Oliva G. Radiomic analysis of the optic nerve at the first episode of acute optic neuritis: an indicator of optic nerve pathology and a predictor of visual recovery? Radiol Med. 2021;126(5):698-706. https://doi.org/10.1007/s11547-020-01318-4 Epub 2021 Jan 3. PMID: 33392980.

61. Kirienko M, Ninatti G, Cozzi L, Voulaz E, Gennaro N, Barajon I, et al. Computed tomography (CT)-derived radiomic features differentiate prevascular mediastinum masses as thymic neoplasms versus lymphomas. Radiol Med. 2020;125(10):951-60. https://doi.org/10.1007/s11547-020-01188w Epub 2020 Apr 18. PMID: 32306201.

62. Hu HT, Shan QY, Chen SL, Li B, Feng ST, Xu EJ, et al. CT-based radiomics for preoperative prediction of early recurrent hepatocellular carcinoma: technical reproducibility of acquisition and scanners. Radiol Med. 2020; 125(8):697-705. https://doi.org/10.1007/s11547-020-01174-2 Epub 2020 Mar 21. PMID: 32200455

63. Farchione $A$, Larici AR, Masciocchi C, Cicchetti G, Congedo MT, Franchi $P$, et al. Exploring technical issues in personalized medicine: NSCLC survival prediction by quantitative image analysis-usefulness of density correction of volumetric CT data. Radiol Med. 2020;125(7):625-35. https://doi.org/10.1007/ s11547-020-01157-3 Epub 2020 Mar 3. PMID: 32125637.

64. Rossi F, Bignotti B, Bianchi L, Picasso R, Martinoli C, Tagliafico AS. Radiomics of peripheral nerves MRI in mild carpal and cubital tunnel syndrome. Radiol Med. 2020;125(2):197-203. https://doi.org/10.1007/s11547-019-01110-z Epub 2019 Nov 26. PMID: 31773457.

65. Paoletti M, Muzic SI, Marchetti F, Farina LM, Bastianello S, Pichiecchio A. Differential imaging of atypical demyelinating lesions of the central nervous system. Radiol Med. 2021. https://doi.org/10.1007/s11547-021-01334-y Epub ahead of print. PMID: 33486703.

66. Fusco R, Granata V, Petrillo A. Introduction to Special Issue of Radiology and Imaging of Cancer. Cancers (Basel). 2020;12(9):2665. https://doi.org/10.3390/ cancers12092665 PMID: 32961946; PMCID: PMC7565136.

67. Nardone V, Reginelli A, Guida C, Belfiore MP, Biondi M, Mormile M, et al. Delta-radiomics increases multicentre reproducibility: a phantom study. Med Oncol. 2020 Mar 31;37(5):38. https://doi.org/10.1007/s12032-020-01359-9.
68. Ametrano G, Riccitiello F, Amato M, Formisano A, Muto M, Grassi R, et al. Analisi anatomiche di molari mandibolari pre- e post-strumentazione con Reciproc mediante $\mu \mathrm{TC}[\mu \mathrm{CT}$ analysis of mandibular molars before and after instrumentation by Reciproc files]. Recenti Prog Med. 2013;104(7-8):420-4. https://doi.org/10.1701/1315.14589.

69. Reginelli A, Capasso R, Petrillo M, Rossi C, Faella P, Grassi R, et al. Looking for Lepidic component inside invasive adenocarcinomas appearing as CT solid solitary pulmonary nodules (SPNs): CT Morpho-Densitometric features and 18-FDG PET findings. Biomed Res Int. 2019;2019:7683648.

\section{Publisher's Note}

Springer Nature remains neutral with regard to jurisdictional claims in published maps and institutional affiliations.
Ready to submit your research? Choose BMC and benefit from:

- fast, convenient online submission

- thorough peer review by experienced researchers in your field

- rapid publication on acceptance

- support for research data, including large and complex data types

- gold Open Access which fosters wider collaboration and increased citations

- maximum visibility for your research: over $100 \mathrm{M}$ website views per year

At BMC, research is always in progress.

Learn more biomedcentral.com/submissions 